\title{
LA EVALUACIÓN DEL DESEMPEÑO DOCENTE: UNA PRÁCTICA DE MEJORA CONTINUA EN LA UNIVERSIDAD ESTATAL PENÍNSULA DE SANTA ELENA.
}

\section{EVALUATION OF THE TEACHING PERFORMANCE: A PRACTICE OF CONTINUOUS IMPROVEMENT AT STATE UNIVERSITY OF SANTA ELENA PENINSULA}

\author{
Aníbal Javier Puya Lino, MSc. Docente-Coordinador de Currículo, Titulación y Capacitación \\ UPSE. apuya@upse.edu.ec \\ Carlos Manuel Castillo Gallo, MSc. Docente-Director de Postgrado UPSE. ccastillo@upse.edu.ec
}

\section{RESUMEN}

El objetivo fundamental de este ensayo es socializar con la comunidad académica que ejerce la docencia superior, los aspectos relacionados con la evaluación del desempeño docente desarrollada en la Universidad Estatal Península de Santa Elena, desde un enfoque teórico práctico. El objetivo general de este artículo es valorar la importancia que tiene la evaluación del desempeño docente, como un proceso de mejora continua en la enseñanza-aprendizaje, la investigación y la vinculación con la comunidad. Todos estos planteamientos han suscitado las siguientes interrogantes que se intenta dar respuesta en el presente reporte científico: ¿Qué se entiende por evaluación del desempeño docente?, ¿cuál es la importancia de la evaluación del desempeño docente?, ¿cuáles son los modelos de evaluación del desempeño docente que se aplican con mayor frecuencia en las universidades latinoamericanas?, ¿qué relación existe entre en el modelo de evaluación del desempeño docente establecido en el Reglamento de Carrera y Escalafón del Profesor e Investigador del Sistema de Educación Superior y el modelo de la UPSE?, ¿cómo se realiza la evaluación del desempeño docente en la UPSE?. Esta investigación ha sido realizada desde un enfoque cualitativo y en la misma se han utilizado los métodos hermenéuticos y fenomenológicos.

Palabras clave: Evaluación del desempeño docente, Reglamento de Carrera y Escalafón del Profesor e Investigador del Sistema de Educación Superior del Ecuador, Pares académicos, Directivos, Mejora continua, autoevaluación, coevaluación y heteroevaluación.

\section{ABSTRACT}

The main objective of this essay is to socialize with the academic community exerted teaching in higher education, issues related to teacher performance assessment developed in the Santa Elena Peninsula State University, from a theoretical and practical approach. The overall objective of this article is to assess the importance of teacher performance assessment as a process of continuous improvement in teaching and learning, research and community outreach. All these approaches have raised the following questions that attempt to answer in this scientific report: What is meant by teacher performance assessment ?, what is the importance of teacher performance assessment ?, what are the assessment models teacher performance applied more frequently in Latin American universities ?, what is the relationship between the model of teacher evaluation provided for in Regulation Career and Pay Scale Research Professor of higher Education System and model UPSE?, how teacher performance assessment is carried out in the UPSE ?. This research was conducted from a qualitative approach and the same have been used hermeneutical and phenomenological methods.

Keywords: Teacher performance assessment, regulation 'Career and Professor and Research System of Higher Education of Ecuador, academic peers, Managers, Continuous improvement, self-assessment, peer assessment and hetero.

Recibido: 30 de noviembre de 2016

Aceptado: 14 de mayo de 2017

Publicado: 30 de junio de 2017 


\section{INTRODUCCIÓN}

El siglo XXI plantea una serie de desafíos y requerimientos a la educación superior relacionados con la calidad, mejoramiento continuo y pertinencia de las funciones sustantivas de la universidad: docencia, investigación, y vinculación con la colectividad. La educación en la actualidad se configura como un proceso complejo, multidimensional e incierto. Además en ella intervienen muchos factores que la condicionan, ya sea a nivel curricular o extracurricular.

En el ámbito curricular, uno de sus elementos es la evaluación que está integrada por muchos componentes, entre ellos la evaluación del aprendizaje, el rendimiento escolar, la evaluación del desempeño docente, las técnicas e instrumentos de evaluación, etc.

Para la realización de la evaluación del desempeño docente, todas las instituciones públicas y privadas de educación superior del Ecuador aplican el modelo propuesto por el Consejo de Educación Superior (CES), establecido en el Reglamento de Carrera y Escalafón del Profesor e Investigador del Sistema de Educación Superior (codificado el 11 Mayo del 2016), Art. 78, el cual propone un modelo basado en tres componentes: Autoevaluación, Coevaluación y Heteroevaluación.

La Universidad Estatal Península de Santa Elena (UPSE), ha venido aplicando el modelo de evaluación de desempeño docente, durante cada uno de los niveles o semestres, al inicio de cada semestre se revisan y actualizan los instrumentos de evaluación por parte de la Comisión Integral de Evaluación, y en lo posterior socializar con los Decanos, Directores de Carrera y docentes de este centro de educación superior. El objetivo que persigue la UPSE, es fortalecer el cuadro docente considerando la evaluación de desempeño que oriente al cumplimiento de los resultados de aprendizaje y la calidad de la Educación Superior.

\section{Evaluación del desempeño docente}

La evaluación del desempeño docente es un proceso que valora las actividades y acciones que realizan los docentes en su ejercicio profesional, con el propósito de buscar el mejoramiento continuo de la calidad de los procesos y productos de la enseñanza y el aprendizaje. Evaluar el desempeño del docente, es un proceso por medio del cual se busca emitir juicios valorativos sobre el cumplimiento de sus responsabilidades en la enseñanza-aprendizaje y desarrollo de sus estudiantes, previo seguimiento de sus acciones, de los avances alcanzados con los estudiantes, desarrollo de su área de trabajo y acciones encaminadas en su unidad académica. Dicha evaluación está referida a la idoneidad, la ética y la pedagogía (1) (Jaime, G. Romero, L. Rincón, E. \& Jaime, L. 2008: Evaluación del desempeño docente. Dialnet, Pág. 167-178). La evaluación del desempeño docente se asume como un proceso permanente enmarcado dentro de una concepción de calidad de la educación, enfocado hacia el perfeccionamiento de la docencia en una institución educativa. La utilización de diversas fuentes de información (estudiantes, colegas, jefe inmediato y el mismo docente) permiten identificar de una manera comprehensiva la labor del profesor y, a partir de ellas, establecer políticas de mejoramiento institucional (2) (Vásquez, F. \& Gabalán, J. 2006: Percepciones estudiantiles y su influencia en la evaluación del profesorado. Un caso en la Universidad Autónoma de Occidente,Cali-Colombia.Relieve,v.12, n. 2, pág.219-245.

Las dos consultas anteriores coinciden que la evaluación del desempeño docente, es un proceso sistemático de la obtención de datos válidos y confiables, que busca el perfeccionamiento docente y la calidad de la educación.

\section{Modelos de evaluación de desempeño docente}

En el IV Coloquio de la Red Iberoamericana de Investigadores sobre Evaluación de la Docencia (RIEED), celebrado del 5 al 8 de marzo del 2013 en la Ciudad de Lima, Perú, expusieron los trabajos realizados a lo largo de cinco años, la red conformada por más de 70 investigadores y profesores de trece diferentes países, expresaron los resultados de tres ejes temáticos: "La evaluación de la docencia y las políticas públicas, educativas e institucionales", "Las prácticas exitosas y perspectivas de la evaluación de la docencia en Iberoamérica" y "Las innovaciones en la evaluación de la docencia en el contexto iberoamericano" en el primer eje que interesa por la investigación, se analizan la evaluación docente, aunque no definen claramente los modelos de evaluación en los diferentes países, especialmente en Universidades Públicas, sin embargo por la lectura se determina: México, utiliza el método centrado en los resultados obtenidos, en la opinión de los estudiantes $y$ en otras universidades participantes utilizan, en primer 
lugar el cuestionario de opinión estudiantil (75\%); cuestionario contestado por el jefe (32\%); cuestionario contestado por el docente, autoevaluación (24\%); observación entre colegas $(24 \%)$, portafolios elaborado por el docente $(20 \%)$, otros $(10 \%)$. En Chile, en la mayoría de las 25 universidades, aunque no se menciona un modelo o enfoque de la docencia, un aspecto que se repite es la declaración de formación por competencias y el modelo basado en la opinión de los estudiantes; En su mayoría refieren que los usos son administrativos y de control, más que formativos, en cambio en Colombia, generalmente centrada en los resultados obtenidos, en la opinión de los estudiantes, en México, centrado en los resultados obtenidos, centrado en el aula. \%).(J. Montoya, I. Arbesú, G. Contreras y S. Conzuelo, RIIED, "Evaluación de la docencia universitaria en México, Chile y Colombia: Análisis de experiencias" 2014) Brasil, utiliza el método de Evaluación por resultados (Adolfo Ignacio Calderón* y Rafael Gabriel de Oliveira Junior, Evaluación por resultados y la cultura de la "performatividad": La evaluación docente en la escuela pública del Estado de San Pablo - Brasil). En España, de acuerdo a la lectura, se puede determinar que la evaluación docente, se los hace en función de las competencias (María Jesús Perales Montolío* Jesús M. Jornet Meliá José González-Such, Tendencias en las políticas de formación y evaluación del profesorado en la Educación Superior en España, Universidad de València). En Cuba, la evaluación docente es formativa y la evaluación basada en la opinión de los estudiantes, (Ángel Martín Aguilar Riveroll, Alejandro Carbonell Duménigoy Edith J. Cisneros-Cohernour, La evaluación de la docencia en dos universidades públicas latinoamericanas: Cuba y México). Los sistemas de evaluación, en la Universidad de Yucatan y en el estado de Campeche, concluyen que las dos universidades han alineado la evaluación de la docencia a las características y funciones que favorecen la Secretaría de Educación Pública, que es la que otorga el estímulo (docencia, investigación, tutoría y trabajo colegiado(Graciela Cordero Arroyo* y Edna Luna Serrano El servicio profesional docente en la perspectiva de los sistemas nacionales de formación de profesores y de evaluación. El caso de México" Universidad Autónoma de Baja California).

Son diversos los modelos utilizados en las diferentes universidades latinoamericanas, centrados generalmente en el perfeccionamiento docente y en la mejora de la calidad de la educación superior. Existen varios modelos de evaluación de desempeño docente: (7) Fernández y Coppola, 2013, tomando como referencia los criterios de Valdés Veloz ,2000. Arbesú, 2004, Rueda, 2008 y Jiménez Moreno 2008).

a.- Modelo centrado en el perfil docente, consiste en evaluar el desempeño de los docentes de acuerdo a las características determinadas en su perfil docente, es decir, determinar un conjunto de indicadores que permitan identificar a un buen profesor. Este perfil puede ser determinado tomando como base las percepciones que tienen los estudiantes, profesores y directivos de cómo debería ser el desempeño de un buen profesor.

b.- Modelo centrado en los resultados obtenidos, el énfasis está puesto en la comprobación de los aprendizajes, tasa de graduación, tasa de repitencia, etc., es decir se evalúa el efecto que produce la actividad del docente en el aprendizaje de los estudiantes. En este modelo se evalúa el éxito o fracaso del docente en función de los resultados de aprendizaje en los estudiantes.

c.- Modelo centrado en el comportamiento del docente en el aula, toma como referencia la interacción entre el profesor y los estudiantes; así como también la capacidad del docente para crear un ambiente favorable para el aprendizaje. El método utilizado con mayor frecuencia para aplicar este modelo de evaluación es la observación.

d.- Modelo de la práctica reflexiva, según (Schön, 1992, citado en Calcina, 2013), se fundamenta en una concepción de la enseñanza como una secuencia de episodios de encontrar y resolver problemas, en la cual las capacidades de los profesores crecen continuamente mientras enfrentan, definen y resuelven problemas prácticos (8).

e.- Modelo basado en la opinión de los estudiantes, éste se caracteriza porque los estudiantes evalúan a sus docentes a través de cuestionarios, este modelo se fundamenta en la heteroevaluación. En este modelo es muy importante la opinión que ofrece el estudiante con respecto al desempeño docente de su profesor. Una de las limitaciones de este modelo es que muchas veces interviene negativamente la opinión del alumno con respecto a su profesor, al no tener suficiente información de todas las actividades del docente que le permita evaluarlo de manera justa, objetiva e imparcial. 
f.- Modelo de evaluación a través de pares, consiste en que otro docente de la misma asignatura y nivel académico pueda evaluar el trabajo de su colega. Este modelo se fundamenta en que el criterio del par académico es de un experto conocedor del tema. En síntesis, en este modelo aplica la coevaluación.

Este tipo de evaluación suele realizarse a través de comisiones encargadas de revisión de medios de verificación del desempeño del profesor, observando las clases y haciendo uso de un instrumento para asegurar la objetividad de los juicios que se emiten (rúbricas o escalas gráficas, descriptivas y numéricas) u observaciones con juicios y sugerencias, o bien, a través de entrevistas.

g.- Modelo de autoevaluación, consiste en permitir que el docente realice una reflexión y valoración personal de su propio desempeño docente evidenciando sus fortalezas y limitaciones. El instrumento que generalmente se utiliza es el cuestionario. Este modelo presenta la desventaja en que muchas veces los docentes no realizan una valoración honesta de su desempeño y tienden a auto asignarse una buena calificación.

h.- Modelo de evaluación a través del portafolio, se parte de la idea de que las evidencias, registros, reflexiones, permiten evaluar de una mejor manera el desempeño del docente, ya que en un portafolio se reúnen distintas evidencias, las reflexiones y el pensamiento del docente detrás del trabajo en las clases. Además, permite conocer los recursos con los cuales el docente, asume la enseñanza y las habilidades, que no necesariamente se ven reflejadas en el aula.

Los modelos de evaluación de desempeño docente mencionados anteriormente tienen sus respectivas ventajas y limitaciones, es muy importante combinarlos adecuadamente $y$ adaptarlos al contexto de cada universidad.

\section{Importancia de la Evaluación del Desempeño Docente}

La evaluación del desempeño docente es importante para todas las instituciones educativas que buscan la calidad, con mayor razón en la educación superior. La aplicación de los diferentes instrumentos en la, autoevaluación, coevaluación y heteroevaluación, en el diagnóstico, proceso de desarrollo y la parte final o sumativa, permiten determinar las deficiencias $y$ retroalimentar, mediante acciones y estrategias en la búsqueda de los resultados de aprendizaje establecidos en la asignatura, en el semestre 0 año, en el perfil profesional del estudiante y en el perfeccionamiento docente. La evaluación del desempeño docente permitirá promover acciones didáctico pedagógicas que favorezcan los procesos de aprendizaje de los estudiantes, y el mejoramiento de la formación inicial docente, así como su desarrollo profesional. La evaluación del docente no debe verse como un acto fiscalizador, sino como una forma de fomentar y favorecer su perfeccionamiento (3) (https://educacion.gob.ec/, s/f, parr. 2 y 3). El concepto de evaluación del desempeño docente en el caso de la educación superior, debe estar asociado a la toma de decisiones y a la implementación de los cambios para superar las debilidades detectadas. Por tanto, bajo este supuesto no basta con determinar los problemas y dificultades en la docencia, sino que también es necesario asociar las propuestas de acción para el mejoramiento, la definición y seguimiento de los mecanismos considerados para elevar los niveles de aprendizaje de los estudiantes, en el marco de las nuevas tendencias que en la actualidad se están dando en la educación superior (4) (Centro Interuniversitario de Desarrollo, CINDA, 2007: Evaluación del Desempeño Docente y Calidad de la Docencia Universitaria, Santiago de Chile: Fondo de Desarrollo Institucional del Ministerio de Educación de Chile, pág. 6). La implementación de la evaluación docente implica un importante cambio que afecta las prácticas, creencias y sentimientos de los profesores, por lo que ellos se convierten en los principales actores de la implementación $y$, por lo tanto, resulta imprescindible tomarlos en cuenta a la hora de evaluar la viabilidad de la propuesta (5) (De Vicente, 2002, Marcelo, 1999, y Murillo, 1999: citados en Catalán y González, 2012, p. 98).

La evaluación del profesorado debe ser un factor importante para mejorar la competencia, la actividad y el reconocimiento de la función docente y de esta manera incidir positivamente en el sistema educativo. Para ello, en todo caso, se requiere de la aceptación y la participación comprometida de los docentes en general. Se logrará en la medida en que estos observen este proceso como una ayuda para mejorar su tarea profesional y no como una amenaza a su seguridad y estabilidad laboral (6) (Garrido y Fuentes, 2008: La evaluación docente, Un aporte a la reconstrucción de prácticas pedagógicas más efectivas. Revista 
Iberoamericana de Evaluación Educativa, Pág. 126-136).

La importancia de la evaluación del desempeño docente se ve reflejada en el mejoramiento continuo de los procesos de enseñanzaaprendizaje; la mejora continua de los docentes; la selección de los temas de capacitación, formación de los profesores; la toma de decisiones para resolver los problemas identificados en la práctica educativa, en la investigación y en la vinculación con la colectividad.

\section{La evaluación del desempeño docente desde la normativa ecuatoriana.}

El Estado garantizará al personal docente en todos los niveles y modalidades, estabilidad, actualización, formación continua y mejoramiento pedagógico y académico; una remuneración justa, de acuerdo a la profesionalización, desempeño y méritos académicos. La ley regulará la carrera docente y el escalafón; establecerá un sistema nacional de evaluación del desempeño y la política salarial en todos los niveles. Se establecerán políticas de promoción, movilidad y alternancia docente (9) (Asamblea Nacional del Ecuador, 2008: Constitución de la República del Ecuador, pág. 162. Recuperado el 10 de marzo de

2016.

http://www.asambleanacional.gov.ec/documen tos/constitucion de bolsillo.pdf.

Los profesores de las instituciones del sistema de educación superior serán evaluados periódicamente en su desempeño académico (...) (10). Asamblea Nacional del Ecuador, 2008: Constitución de la República del Ecuador, pág. 162.http://www.asambleanacional.gov.ec/docu mentos/constitucion de bolsillo.pdf.

La evaluación integral del desempeño se aplicará a todo el personal académico de las instituciones de educación superior, públicas y particulares. La evaluación integral de desempeño abarca las actividades de docencia, investigación, y dirección o gestión académica" (11) (Consejo de Educación Superior. Reglamento de Carrera y Escalafón del profesor e investigador del Sistema de Educación Superior. Recuperado en 11 de marzo de 2016, (pág. 43 de http://www.ces.gob.ec/descargas/ley-organicade-educacion-superior). La normativa ecuatoriana establece como fin único la calidad de la educación superior y para ello, determina la evaluación de desempeño como una herramienta indispensable y los presupuestos que cada universidad deben asignar para la capacitación y becas del personal docente, para lograr conseguirlo.

La evaluación del desempeño docente es un proceso institucional y sistemático que se aplica al profesor - investigador en las funciones asignadas en el periodo académico, como una forma de mejorar los niveles de aprendizaje en el marco del aseguramiento de la calidad de la Universidad Estatal Península de Santa Elena (12) (Universidad Estatal Península de Santa Elena, 2015: Reglamento de Evaluación del Desempeño Docente, pág. 2)

El proceso de evaluación del desempeño docente de acuerdo al Reglamento de Carrera y Escalafón del Profesor e Investigador del Sistema de Educación Superior, del Consejo de Educación Superior (CES), se realiza mediante tres criterios que se interrelacionan $y$ complementan: autoevaluación, coevaluación y heteroevaluación. La autoevaluación es la evaluación que el personal académico realiza periódicamente sobre su trabajo y su desempeño académico. La coevaluación es la evaluación que realizan pares académicos y, la heteroevaluación es la evaluación que realizan los estudiantes sobre el proceso de aprendizaje impartido por el personal académico.

\section{El proceso de evaluación del desempeño docente aplicado en la UPSE}

Aunque anteriormente se realizaba la evaluación considerando los tres criterios, en el periodo académico 2015 - I, se conformó la Comisión Integral de Evaluación del Desempeño Docente encargada de realizar los ajustes de reforma al Reglamento y de diseñar el proceso técnico instrumental de presentación, análisis e interpretación de la información y dar legitimidad a los resultados y las decisiones que de ellos se deriven, los mismos que fueron aprobados por el Consejo Superior Universitario de conformidad a la normatividad oficial establecida.

Con la nueva reglamentación, la evaluación del desempeño docente se realiza a través de los tres criterios mencionados anteriormente, y se establecen con la finalidad de obtener información para medir la productividad académica centrada en las funciones de docencia, vinculación con la colectividad e investigación. La evaluación del desempeño docente está asociada a la toma de decisiones y a la implementación de los cambios para superar las debilidades detectadas. Por tanto, bajo este supuesto, no basta con determinar los problemas y dificultades en la docencia, sino que también es necesario asociar las 
propuestas de acción para el mejoramiento, la definición y seguimiento de los mecanismos considerados para elevar los niveles de aprendizaje de los estudiantes, en el marco de las nuevas tendencias que en la actualidad se están dando en la educación superior.

\section{Objetivos de la evaluación del desempeño docente}

Los objetivos de la evaluación son (14):

a) Diagnosticar el trabajo y desempeño académico de los profesores, mediante la aplicación de técnicas e instrumentos de recopilación de información, que determinen el grado de satisfacción de la comunidad universitaria.

b) Planificar y ejecutar programas de capacitación y actualización pedagógica, en función del diagnóstico para el fortalecimiento de la calidad de gestión en los diferentes ámbitos del desempeño académico.

c) Mejorar el desempeño del profesor en base a los resultados de la evaluación para retroalimentar los procesos académicos.

d) Proveer a las autoridades de la Universidad Estatal Península de Santa Elena la información para la toma de decisiones relacionadas con la planificación de actividades de su personal académico.

f) Promover la cultura de evaluación en un ambiente de interacción entre actores que ejercen la libertad de cátedra con responsabilidad académica.

\section{METODOLOGÍA}

Los actores del proceso de evaluación de desempeño están conformados por Rector(a), Vicerrector(a) Académico(a), Decanos, Directores de carrera, Pares académicos, Profesores(a) y Estudiantes (15), cada uno de los actores evaluará de acuerdo a la sucesión y los directores de carrera evaluarán a los docentes, de acuerdo a la tabla 1. La evaluación del desempeño está en función del distributivo de carga horaria del docente, aprobada por el Consejo Superior Universitario, y se realiza considerando tres criterios que se interrelacionan y se complementan entre sí, la evaluación, autoevaluación y heteroevaluación, con la única finalidad de lograr conseguir la profesionalización de los docentes y los resultados de aprendizaje de la carrera.

La Coevaluación.- Está conformada por la evaluación de los Directivos y pares académicos con diferentes valoraciones en función de la tabla 1 y del distributivo de carga horaria de cada docente.

Directivos.- El director de la carrera, tiene cuatro instrumentos de evaluación. La evaluación de desempeño inicia antes del comienzo del ciclo académico, con la realización de actualización pedagógica, basado en el análisis de los resultados de los informes de evaluación de desempeño docente del ciclo anterior programada por el Vicerrectorado Académico. Posteriormente los Directores de Carrera realizan seminarios talleres para la elaboración y análisis de los Sílabos y planes de clases.

Al Inicia del ciclo, los docentes se encargan de crear los recursos necesarios en la Plataforma virtual, (Recursos de aprendizaje, el portafolio electrónico, y demás instrumentos curriculares), los cuales son revisados por los pares académicos (comisión áulica) y directivos.

La comisión áulica en el transcurso del ciclo académico, realiza las siguientes actividades: las observaciones de las clases de los docentes, y hace conocer los resultados, con sus respectivas recomendaciones didácticos pedagógicas. Además hace seguimiento a las diferentes actividades diseñadas por los docentes en la plataforma. El instrumento utilizado en la coevaluación es una ficha de evaluación áulica.

Heteroevaluación.- Los docentes son evaluados por sus estudiantes, y tiene una valoración de 40 puntos, lo realizan vía online a través de la plataforma virtual en función de la normativa vigente, antes de la culminación de los ciclos académicos, proceso que se lo realiza aplicando una encuesta de 20 ítems, con escala numérica; en la cual se evalúan tres componentes: Planificación Ejecución y Evaluación del proceso de la clase.

La autoevaluación, la dirección de la carrera en el proceso de desarrollo del ciclo, solicita con un instrumento enviado por correo las respuestas a un sinnúmero de preguntas que tienen una valoración de 15 puntos. 
Tabla 1

Ponderaciones por componentes

\begin{tabular}{|c|c|c|c|c|c|c|}
\hline \multirow{3}{*}{ COMPONENTES } & \multirow{3}{*}{$\begin{array}{l}\text { AUTOEVA- } \\
\text { LUACIÓN }\end{array}$} & \multicolumn{3}{|c|}{ COEVALUACIÓN } & \multirow{3}{*}{$\begin{array}{c}\text { HETERO- } \\
\text { EVALUACIÓN }\end{array}$} & \multirow{3}{*}{ TOTAL } \\
\hline & & \multirow{2}{*}{ DIRECTIVO } & \multicolumn{2}{|c|}{ PARES } & & \\
\hline & & & FUNCIÓN & COMISIÓN & & \\
\hline Docencia & 15 & 20 & 0 & 25 & 40 & 100 \\
\hline $\begin{array}{l}\text { Docencia-Vinculación } \\
\text { con la colectividad }\end{array}$ & 15 & 20 & 12,5 & 12,5 & 40 & 100 \\
\hline Investigación & 15 & 35 & 25 & 25 & 0 & 100 \\
\hline $\begin{array}{l}\text { Dirección o Gestión } \\
\text { Académica }\end{array}$ & 15 & 40 & 15 & 15 & 15 & 100 \\
\hline
\end{tabular}

Fuente: Reforma del Reglamento de Evaluación del Desempeño Docente de la UPSE (2015)

Los instrumentos de: autoevaluación, han sido actualizados y aplicados considerando el desempeño del ejercicio docente, en el que se consideran, actividades de planificación, proceso de aprendizaje y evaluación de la clase; mientras que en los instrumentos de coevaluación (directivo y comisión) se evalúan las funciones de gestión, investigación y vinculación con la colectividad asignadas a los docentes a tiempo completo, en el caso de gestión, los jefes inmediatos evalúan; vinculación con la colectividad e investigación, los directores de estas dependencias son los que evalúan.

Para realizar este proceso se realizó un plan de ejecución, el mismo que fue aprobado por el Vicerrectorado Académico y contenía, desde la actualización del reglamento, instrumentos de evaluación, ponderación, componentes, hasta la entrega de resultados. Esta planificación fue socializada a todos los involucrados en una sesión de trabajo que permitió la retroalimentación inmediata de parte de los docentes.

Para la tabulación de los datos, se ha implementado un sistema informático que permite ingresar los resultados obtenidos de la autoevaluación y coevaluación, mientras que los datos de la heteroevaluación son procesados en cada una de las dependencias académicas y, entregados a la Comisión para poder consolidarlos y ponderarlos de acuerdo al componente de cada docente.

Los informes preliminares y finales por docente son enviados a los Directores de Carreras para que se entreguen a los docentes $y$, de ser el caso, atender las solicitudes de apelaciones.

La valoración del trabajo y desempeño de los profesores se expresa cualitativa y cuantitativamente como se indica en el cuadro siguiente:
Tabla 2

Escala valorativa

\begin{tabular}{|l|c|}
\hline $\begin{array}{c}\text { Evaluación } \\
\text { cualitativa }\end{array}$ & $\begin{array}{c}\text { Evaluación } \\
\text { cuantitativa }\end{array}$ \\
\hline Excelente & De 95 a 100 \\
\hline Muy bueno & De 85 a 94 \\
\hline Bueno & De 70 a 84 \\
\hline Regular & De 60 a 69 \\
\hline Insuficiente & De 0 a 59 \\
\hline
\end{tabular}

Fuente: Reforma del Reglamento de Evaluación del Desempeño Docente de la UPSE

\section{Resultados de la Evaluación de desempeño docente en el periodo 2015-II}

Los resultados obtenidos de la evaluación realizada a 186 docentes, la tabla 3 identifica a 27 docentes que tienen una calificación de excelente, 118 muy bueno y 37 bueno, 2 regulares y 2 insuficientes, de los buenos adelante suman 41 , equivalente al $22 \%$, que debe establecerse las deficiencias y tratar en lo posible que en la próxima evaluación mejoren su calificación y sus resultados de aprendizaje.

Tabla 3

Cualificación de los docentes de la UPSE del 2015-2

\begin{tabular}{|l|c|}
\hline Cualificación & $\mathbf{N}^{\circ}$ Docentes \\
\hline Excelente & $\mathbf{2 7}$ \\
\hline Muy bueno & $\mathbf{1 1 8}$ \\
\hline Bueno & $\mathbf{3 7}$ \\
\hline Regular & $\mathbf{2}$ \\
\hline Insuficiente & $\mathbf{2}$ \\
\hline Total & $\mathbf{1 8 6}$ \\
\hline
\end{tabular}

Fuente: Informe de la UNOPAC

Los promedios de las calificaciones de la evaluación de desempeño docente por componentes, se muestran en la tabla 4 . De acuerdo a la tabla 2 el promedio general es muy 
bueno y que con el mejoramiento continua en la siguiente fase pasarían a ser excelentes.

Tabla 4

Promedio de calificaciones por componentes del 2015-2

\begin{tabular}{|l|c|}
\hline Componentes & Promedio calif. \\
\hline Investigación & 88,53 \\
\hline Dirección o Gestión académica & 87,40 \\
\hline Vinculación con la colectividad & 87,27 \\
\hline Docencia & 86,45 \\
\hline Promedio general & 87,39 \\
\hline
\end{tabular}

Tabla 5

Resultados de la Evaluación por criterio y componentes en 2015-2

\begin{tabular}{|c|c|c|c|c|c|c|}
\hline \multirow{3}{*}{ COMPONENTES } & \multirow{3}{*}{$\begin{array}{l}\text { AUTOEVALUA- } \\
\text { CIÓN }\end{array}$} & \multicolumn{3}{|c|}{ COEVALUACIÓN } & \multirow[b]{3}{*}{$\begin{array}{l}\text { HETERROEVALUA- } \\
\text { CIÓN }\end{array}$} & \multirow{3}{*}{ TOTAL } \\
\hline & & \multirow[b]{2}{*}{$\begin{array}{l}\text { DIRECTI- } \\
\text { VOS }\end{array}$} & \multicolumn{2}{|c|}{ PARES } & & \\
\hline & & & $\begin{array}{l}\text { FUN- } \\
\text { CIÓN }\end{array}$ & $\begin{array}{l}\text { COMI- } \\
\text { SIÓN }\end{array}$ & & \\
\hline Docencia & 14,07 & 17,44 & 0 & 23,5 & 32,39 & 87,41 \\
\hline $\begin{array}{l}\text { Docencia-Vinculación } \\
\text { con la colectividad }\end{array}$ & 14,10 & 17,69 & 10,86 & 11,88 & 33,02 & 87,56 \\
\hline Investigación & 14,12 & 31,59 & 21,86 & 23,6 & & 90,99 \\
\hline $\begin{array}{l}\text { Dirección o Gestión } \\
\text { académica }\end{array}$ & 13,85 & 36,84 & 14,13 & 14,48 & 13 & 92,3 \\
\hline
\end{tabular}

Fuente: Informe de la UNOPAC

Con la finalidad de mejorar los procesos de enseñanza aprendizaje, el Vicerrectorado Académico convoca a los docentes y directores al seminario taller, en el cual se establecen 5 grupos, para revisar los temas; Diseño curricular y Sílabo, componentes del plan de clase, evaluación de aprendizaje, evaluación de desempeño docente y articulación de docencia, investigación y vinculación.

Uno de los grupos es el de evaluación desempeño docente, en el cual hacen ciertas observaciones los docentes y consideran que deben realizar los ajustes en los instrumentos correspondientes para lograr conseguir una evaluación docente acorde con la realidad.

\section{CONCLUSIONES}

- La evaluación del desempeño docente es un proceso continuo, integral y sistémico que permite obtener información acerca de las funciones inherentes a los docentes en el ámbito académico, de gestión, investigación y vinculación con el propósito de tomar decisiones en la planificación institucional, los programas de capacitación y formación docente, el mejoramiento continuo del proceso de enseñanza aprendizaje, la adaptación e innovación curricular en relación de los problemas, necesidades y expectativas de los docentes.

- La evaluación del desempeño docente no debe tener carácter punitivo ni
Fuente: Informe de la UNOPAC

Los resultados de la evaluación de desempeño por criterio y componentes, se expresan en la tabla 5, los mismos que los Directores, deben revisar los instrumentos y ponderar las capacitaciones que se requieren para mejorar los resultados de aprendizaje de la carrera y la institución. 
en la evaluación del profesorado. Un caso en la Universidad Autónoma de Occidente, Cali-Colombia. RELIEVE, v. 12, n. 2, pág. 219-220-245.

http://www.uv.es/RELIEVE/v12n2/RELIEVE v12n2 3.htm

3.- En la página web oficial del Ministerio de Educación del Ecuador (s/f) (parr. 2 y 3).

4.- Centro Interuniversitario de Desarrollo (CINDA, 2007). Evaluación del Desempeño Docente y Calidad de la Docencia Universitaria, Santiago de Chile: Fondo de Desarrollo Institucional del Ministerio de Educación de Chile.(pág. 6)

5.- De Vicente, 2002, Marcelo, 1999, y Murillo, 1999; citados en Catalán y González, 2012(p. 98)

6.- Garrido y Fuentes (2008) La evaluación docente: Un aporte a la reconstrucción de prácticas pedagógicas más efectivas. Revista Iberoamericana de Evaluación Educativa, 126-136.

7.- Fernández y Coppola (2013), tomando como referencia los criterios de Valdés Veloz (2000), Arbesú (2004), Rueda (2008) y Jiménez Moreno (2008)

8.- (Schön,1992, citado en Calcina, 2013)(pág. 25)

9.- Asamblea Nacional del Ecuador (2008). Constitución de la República del Ecuador, pág. 162.
Recuperado el 10 de marzo de 2016, de http://www.asambleanacional.gov.ec/docu mentos/constitucion de bolsillo.pdf.

10.-Consejo de Educación Superior. Ley Orgánica de Educación Superior(LOES2010), Art. 55 (p. 24). Recuperado en 11 de marzo de 2016, de http://www.ces.gob.ec/descargas/leyorganica-de-educacion superior.

11.-Consejo de Educación Superior. Reglamento de Carrera y Escalafón del profesor e investigador del Sistema de Educación Superior. Recuperado en 11 de marzo de 2016,(pág. 43 de http://www.ces.gob.ec/descargas/leyorganica-de-educacion-superior.

12.- Universidad Estatal Península de Santa Elena (UPSE), El reglamento de Evaluación del Desempeño Docente, 2015, I (pág. 2)

13.- (CES), El Reglamento de Carrera y Escalafón del Profesor e Investigador del Sistema de Educación Superior.

14.- Universidad Estatal Península de Santa Elena, 2015 (UPSE), El reglamento de Evaluación del Desempeño Docente.

15.- Universidad Estatal Península de Santa Elena (UPSE), Reglamento de Evaluación del Desempeño Docente, 2015, I (art. 12).

16.- Red Iberoamericana de Investigadores sobre Evaluación de la Docencia (RIEED), celebrado del 5 al 8 de marzo del 2013 